\title{
Big Cloud Innovation for Sustainable Wireless Management
}

\author{
Chan-Yun Yang ${ }^{1} \cdot$ Muhammad Sharif $^{2} \cdot$ Sri Devi Ravana ${ }^{3} \cdot$ Anandakumar Haldorai $^{4}$ \\ Published online: 19 February 2021 \\ (c) The Author(s), under exclusive licence to Springer Science+Business Media, LLC part of Springer Nature 2021
}

Big data and Cloud Computing paradigm poses new challenges to the communication technology as numerous heterogeneous objects will need to be connected and supports a wide variety of applications. With the recent growth of Big Cloud computing based applications the usage of big data services is increasing exponentially. Big Cloud is a promising technology that enables intelligent data collection and processing on various sustainable cloud applications. The next generations of big cloud emerging application domains are approaching existing principles and technologies to their limits. Opportunely, Cloud development community is responding to these challenges with new theories and technologies capable of handling increasing dynamism, context-awareness and large-scale adaptation and evolution of software, development and environments.

The wireless network communication for the big data and cloud system would enable maximum utilization of resources, causing an enhancement in the wireless communication system efficiency, by sharing the infrastructure among multiple applications or service provisioning. Cloud and bigdata access can leverage on the sustainable wireless network offerings and gain complete solution over the network to strengthen wireless communication services. Big cloud techniques help in establishing flexible framework over the infrastructure, thereby assisting in optimizing the operations in a more fruitful manner.

The digital revolution led by the big cloud wireless management is already reshaping several traditional business sectors. In this issue, we discuss how data science for sustainable cloud applications, cloud platform for privacy preserving data science, social data relationship ranking on data grid, data grid applications for cognitive communication, machine

Chan-Yun Yang

cyyang@mail.ntpu.edu.tw

Muhammad Sharif

muhammadsharifmalik@yahoo.com

Sri Devi Ravana

sdevi@um.edu.my

Anandakumar Haldorai

anandakumar.h@sece.ac.in

1 College of Electrical Engineering and Computer Science, National Taipei University, Taipei,

Taiwan

2 COMSATS University Islamabad, Wah Campus, Wah Cantt, Pakistan

3 University of Malaya, Kuala Lumpur, Malaysia

4 Sri Eshwar College of Engineering, Coimbatore, Tamil Nadu, India 
learning and big data in cloud development, next generation wireless communication and smart city initiative and cloud services/infrastructures can alleviate some real environmental problems. This issue focuses high quality research papers that address significant and new big cloud and related system development issues in the emerging sustainable application domains.

We would like to thank the Editor-in-Chief Professor Ramjee Prasad, Ms. Angelina Wagner Journal Development Manager, Mr. Meertinus Faber Project Coordinator of Production at Springer Nature, and Mr. Joseph Ian Reyes Springer's Journal's Editorial Office Assistant for their support in this special issue. We thank all the authors for their excellent contributions and reviewers for their treasurable comments. We anticipate that this issue will open new entrance for further research and technology improvements in this important area.

Publisher's Note Springer Nature remains neutral with regard to jurisdictional claims in published maps and institutional affiliations.
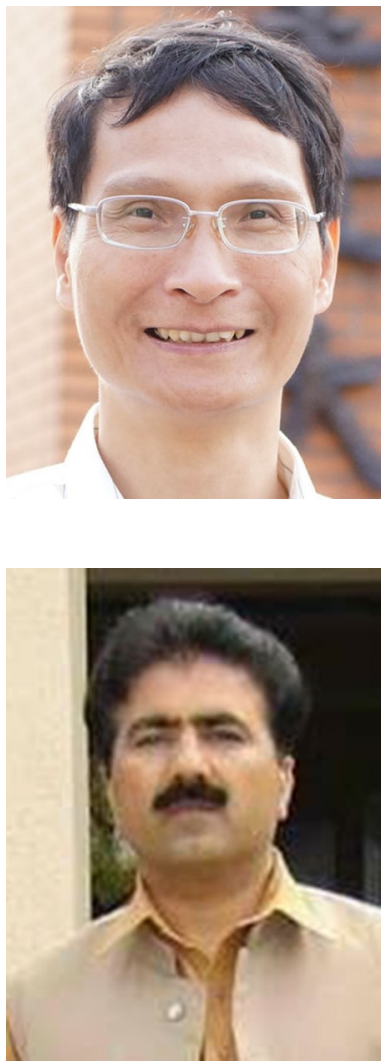

Dr. Chan-Yun Yang is the Director of the Intelligent Modeling and Control Lab and Professor with the Department of Electrical Engineering, College of Electrical Engineering and Computer Science, National Taipei University, Taiwan. His career in robotics and artificial intelligence research has spanned many countries. He was awarded a Ph.D. degree from the Department of Bio-Industrial Mechatronics Engineering, National Taiwan University. After obtaining his Ph.D. degree he became a Research Fellow at the Keio-NUS CUTE Centre, which is a collaborative research center with locations in both the National University of Singapore (NUS) and Keio University in Japan. He is also actively serving several robotics and AI related journals and conferences as Editorial Board member, Organizing Committee member, workshop organizer and reviewer.

Dr. Muhammad Sharif (IEEE Senior Member) is an Associate Professor at COMSATS University Islamabad, Wah Cantt Pakistan. He has completed his Ph.D. in Image Processing from COMSATS Institute of IT, Islamabad in 2013. He got his M.S. (CS) degree from the same institute in 2007 and MCS degree from Quaid-e-Azam University, Islamabad Pakistan in 1995. He has more than 150 research publications in IF, SCI and ISI journals as well as in national and international conferences and obtained 100+ Impact Factor. His research interests include Medical Imaging, Biometrics, Computer Vision, Machine Learning and Agriculture/Plants imaging. 


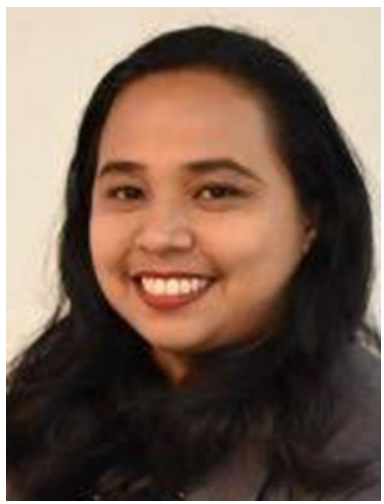

Dr. Sri Devi Ravana received the bachelor's degree in information technology from the National University of Malaysia, in 2000, the master's degree in software engineering from the University of Malaya, Malaysia, in 2001, and the Ph.D. degree from the Department of Computer Science and Software Engineering, The University of Melbourne, Australia, in 2012. She is currently an Associate Professor at the Department of Information Systems, University of Malaya. Her research interests include information retrieval heuristics, text indexing, data analytics, and data mining. She received a couple of best paper awards in international conferences within the area of information retrieval.

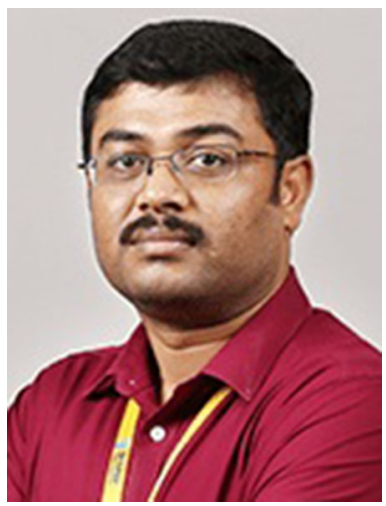

Dr. Anandakumar Haldorai Professor (Associate) and Research Head in Department of Computer Science and Engineering, Sri Eshwar College of Engineering, Coimbatore, Tamil Nadu, India. He has received his Master's in Software Engineering from PSG College of Technology, Coimbatore, Ph.D. in Information and Communication Engineering from PSG College of Technology under, Anna University, Chennai. His research areas include Cognitive Radio Networks, Mobile Communications and Networking Protocols. He has authored more than 118 research papers in reputed International Journals and IEEE, Springer Conferences. He has authored 11 books and many book chapters with reputed publishers such as Springer and IGI. He has served as Editor in Chief in Keai-Elsevier IJIN and reviewer for IEEE, IET, Springer, Inderscience and Elsevier journals. He is also the guest editor of many journals with Wiley, Springer, Elsevier, Inderscience, etc. He has been the General chair, Session Chair, and Panelist in several conferences. He is a senior member of IEEE, MIET, MACM and Fellow member of EAI research group. 